ORIGINAL ARTICLE

\title{
Dyslipidemia and atherogenic index of plasma in gestational diabetes mellitus with vitamin B12 deficiency.
}

\author{
Shehmeen Khan 1 , Sabreena Abbas², Afsheen Abro ${ }^{3}$, Beenish Ghafar Memon ${ }^{4}$, Farhat Sultana ${ }^{5}$, Hafsa Usman Shaikh
}

Article Citation: Khan S, Abbas S, Abro A, Memon BG, Sultana F, Shaikh HU. Dyslipidemia and atherogenic index of plasma in gestational diabetes mellitus with vitamin B12 deficiency. Professional Med J 2022; 29(3):367-371. https://doi.org/10.29309/TPMJ/2022.29.03.6827

\begin{abstract}
Objective: To determine frequency of vitamin B12 deficiency and dyslipidemia and calculate atherogenic index of plasma (AIP) in Gestational Diabetes mellitus (GDM). Study Design: Observational study. Setting: Department of Biochemistry, Liaquat University Jamshoro. Period: January 2018 to December 2019. Material \& Methods: 216 cases of GDM were selected by inclusion criteria. Venous blood sampling collected blood sample that were centrifuged to get sera. Glucose, vitamin B12, and blood lipids were estimated by standard methods. Atherogenic Index of Plasma (AIP) was calculated as log TAG/HDLc ratio. Data was analysed by SPSS 21.0 ver. at $95 \% \mathrm{Cl}(\mathrm{P} \leq 0.05)$. Results: Vitamin B12 deficiency and Dyslipidemia were noted in 151 (69.9\%) and $165(23.61 \%)$ of GDM cases respectively $(P=0.0001)$. Vitamin B12 was found low as $156.62 \pm 82.9 \mathrm{ng} / \mathrm{mL}$. TC (244.07 \pm 49.11$)$, TAGs $(699.4 \pm 249.5)$, and LDLc (137.9 \pm 40.84$)$ were found high and HDLc $(33.13 \pm 5.71)$ was low. High and medium risk AIP was noted in $17(7.87 \%)$ and $29(13.4 \%)$ of GDM cases $(P=0.0001)$. Vitamin $B 12$ proved negative TC $(r=-0.25, P=0.02)$, TAG $(r=-0.23, P=0.02), L D L c(r=-0.79, P=0.0001)$ and positive correlation with HDLc $(r=0.76, P=0.0001)$. Conclusion: Vitamin B12 deficiency and Dyslipidemia were noted in 151 (69.9\%) and 165 (23.61\%) of GDM cases respectively. High and medium risk AIP was noted in 17 (7.87\%) and $29(13.4 \%)$ of GDM cases.
\end{abstract}

Key words: $\quad$ Atherogenic Index, Dyslipidemia, Vitamin B12 Deficiency.

\section{INTRODUCTION}

Gestational diabetes mellitus (GDM) is a metabolic disorder of blood glucose homeostasis characterized by hyperglycemia of variable severity and glucosuria noted first time during pregnancy. ${ }^{1,2} 80-90 \%$ of pregnant women are diagnosed as GDM for the first time in pregnancy. Predisposing factors include high body mass index $\left(>25 \mathrm{~kg} / \mathrm{m}^{2}\right)$, family history, macrosomia, previous GDM, polyhydramnios, and women of South East Asia, Black Caribbean, and Middle East, etc. Other risk factors are the polycystic ovarian syndrome (PCOS), pregnancy induced glucosuria, and pregnancy induced hypertension, congenital anomalies, still births, miscarriage, unexplained fetal deaths, etc. ${ }^{1,2}$ IDF shows $16 \%$ GDM cases with an incidence of $>200,000$ cases annually. However, incidence varies according to the ethnicity, screening programs, and GDM criteria of diagnosis used. Its prevalence is inclining in the low and middle income countries. Insulin resistance is common feature of GDM diagnosed at the end of $2^{\text {nd }}$ trimester. GDM appears when pancreatic $\beta$-cell function is not sufficient to overcome the anti - insulin hormones and insulin resistance. ${ }^{1,2}$ Metabolic derangement of GDM is aggravated by concomitant malnutrition and vitamin deficiencies. As the B -complex vitamins are essential for glucose metabolism ${ }^{3}$, this results in altered levels of blood lipids called the dyslipidemia. Dyslipidemia is defined as elevated blood cholesterol and triglycerides in presence of low HDLc. ${ }^{4}$ Deficiency of vitamin B12 has been associated with dyslipidemia in diabetics. Vitamin B12 is an essential vitamin of B- complex family and its deficiency is well noted in general and diabetic populations. Vitamin B12 is needed for DNA methylation,
1. MBBS, M.Phil, Lecturer, Bilawal Medical College Jamshoro.

2. MBBS, FCPS, Assistant Professor Gynecology and Obstetrics, Liaquat University of Medical \& Health Sciences Jamshoro. 3. MBBS, M.Phil, Lecturer, Bilawal Medical College Jamshoro.

4. MBBS, Mphil, Assistant Professor Gynecology and Obstetrics, Liaquat University of Medical \& Health Sciences Jamshoro.

5. MBBS, FCPS, Senior Registrar, Liaquat University of Medical \& Health Sciences Jamshoro.

6. MBBS, Demonstrator Biochemistry, Bilawal Medical College Jamshoro.

\section{Correspondence Address: \\ Dr. Shehmeen Khan \\ Bilawal Medical College Jamshoro. drshehmeenkhan@gmail.com}

Article received on: Accepted for publication:

$28 / 09 / 2021$ $08 / 12 / 202$ 
lipid metabolisms, cell proliferation, and nuclear division. Its deficiency is linked with dyslipidemia and endothelial dysfunctioning. ${ }^{5}$ Vitamin B12 deficiency is linked with coronary artery disease (CAD), myocardial ischemia, cerebral ischemia, diabetic microvascular complications such as neuropathy. ${ }^{5,6}$ Vitamin B12 deficiency is associated with births defects, brain malformations, defective myelination, polyhydramnios, respiratory distress syndrome, fetal malformations and still births. ${ }^{6,7}$

However, scarcity of research is lagging on the epigenetic effects of vitamin B12 deficiency during pregnancy. ${ }^{8-10}$ The present study was conducted to determine frequency of dyslipidemia and atherogenic index of plasma in Gestational Diabetes mellitus with vitamin $B_{12}$ deficiency.

\section{MATERIAL \& METHODS}

An observational study was conducted at the Department of Biochemistry, Liaquat University of Medical and Health Sciences, Jamshoro. Study was conducted from January 2018 to December 2019. Patients were collected from the Department of Gynecology and Obstetrics, Liaquat University Hospital Jamshoro/Hyderabad. A sample of 216 diagnosed cases of Gestational Diabetes mellitus (GDM) were collected through non-probability purposive technique. GDM was defined according to the American Diabetes Association. ${ }^{8}$ Inclusion criteria were; diagnosed GDM cases, age $20-40$ years, $2-3$ trimester of pregnancy, and single fetus. Already diagnosed cases of DM, metformin therapy, insulin resistance, polycystic ovarian syndrome (PCOS) and those suffering major systemic illness were excluded from study protocol. Volunteers GDM cases were interviewed about purpose of study. They were informed that study will cause no harm to them and their babies. Only those pregnant ladies were included who gave a volunteer consent.

Patients were examined by a senior consultant gynecologist before entry into the study protocol. Patient's data and findings of physical examination were saved in a proforma. Patient's personal data was kept confidential in lockers. Only principal investigator had access to the Biodata and physical examination findings. Study was taken permission from institutional ethics committee (LUMHS/REC/634). Patients handling was according to the "Helsinki's Declaration" for conducting human research. Volunteer GDM cases were asked for venous blood sample collection from a prominent peripheral vein preferably of upper arm. $5 \mathrm{ml}$ venous sample was taken in a sodium fluoride containing glass tubes. Sera were got by centrifuging blood at x3000 rpm for fifteen minutes. Sera were preserved at $-20{ }^{\circ} \mathrm{C}$ temperature in refrigerators. Sera was used for glucose estimation by hexokinase method and vitamin B12 by ELISA method ${ }^{9}$ using commercial kit (Abcam, USA). Blood total cholesterol (TC), triglycerides (TAGs), LDLc and HDLc were detected using Cobas chemistry analyzer. Atherogenic Index of Plasma (AIP) was calculated as log TAG/HDLc ratio. ${ }^{10}$ AIP grading was done as; low risk (- $0.3-0.1)$, medium risk $(0.1-0.24)$ and high risk (>0.24).${ }^{10}$ Results were analysed on SPSS 21.0 ver. Categorical variables were calculated by $\mathrm{CHI}$-square test and continuous variable were calculated by Student's t-test. Output of categorical results was presented as frequency and \% while, continuous output was tabulated as mean+/- SD. Data analysis level of significance was $95 \% \mathrm{Cl}(\mathrm{P} \leq 0.05)$.

\section{RESULTS}

Mean $( \pm S D)$ age, fasting and random blood glucose, vitamin $B_{12}$, total cholesterol (TC), triglycerides (TAGs), low (LDLC) and high density (HDLc) lipoproteins cholesterol of GDM cases is shown in Table-l. Vitamin $B_{12}$ was found low as $156.62 \pm 82.9 \mathrm{ng} / \mathrm{mL}$. TC $(244.07 \pm 49.11)$, TAGs (699.4 \pm 249.5$)$, and LDLc (137.9 \pm 40.84$)$ were found high and HDLc $(33.13 \pm 5.71)$ was low. Vitamin $B_{12}$ deficiency and Dyslipidemia were noted in 151 (69.9\%) and 165 (23.61\%) of GDM cases respectively $(P=0.0001)$. High and medium risk AIP was noted in 17 (7.87\%) and 29 (13.4\%) of GDM cases $(P=0.0001)$. Vitamin $B_{12}$ proved negative TC $(r=-0.25, P=0.02)$, TAG $(r=-0.23$, $P=0.02)$, LDLC $(r=-0.79, P=0.0001)$ and positive correlation with HDLC $(r=0.76, P=0.0001)$ (Figure-1 to 4). 


\begin{tabular}{|l|c|c|}
\hline \multirow{2}{*}{ Variable } & \multicolumn{2}{c|}{ GDM Cases } \\
\hline Age (years) & Mean & SD \\
\hline FBS (mg/dl) & 36.29 & 9.434 \\
\hline RBS (mg/dl) & 136.27 & 35.35 \\
\hline Vitamin B ${ }_{12}(\mathrm{ng} / \mathrm{mL})$ & 254.25 & 65.92 \\
\hline TC $(\mathrm{mg} / \mathrm{dl})$ & 156.62 & 82.95 \\
\hline TAGs $(\mathrm{mg} / \mathrm{dl})$ & 244.07 & 49.11 \\
\hline LDLc $(\mathrm{mg} / \mathrm{dl})$ & 699.46 & 249.53 \\
\hline HDLc $(\mathrm{mg} / \mathrm{dl})$ & 137.97 & 40.84 \\
\hline
\end{tabular}

TC- total cholesterol

GDM - gestational diabetes mellitus

Table-I. Biochemical findings in GDM cases. $(n=216)$

\begin{tabular}{|c|c|c|c|c|}
\hline & \multicolumn{2}{|c|}{ GDM Cases } & \multirow{2}{*}{$\mathrm{X}^{2}$-Value } & \multirow{2}{*}{ P-Value } \\
\hline & Frequency & $\%$ & & \\
\hline Yes & 151 & 69.9 & \multirow{3}{*}{107.00} & \multirow{3}{*}{0.0001} \\
\hline No & 65 & 30.09 & & \\
\hline Total & 216 & 100 & & \\
\hline
\end{tabular}

Table-II. Vitamin $B_{12}$ deficiency in GDM cases $(n=216)$

\begin{tabular}{|c|c|c|c|c|}
\hline & \multicolumn{2}{|c|}{ GDM Cases } & \multirow{2}{*}{$\mathrm{X}^{2}$-Value } & \multirow{2}{*}{ P-Value } \\
\hline & Frequency & $\%$ & & \\
\hline Yes & 51 & 23.61 & \multirow{3}{*}{353.00} & \multirow{3}{*}{0.0001} \\
\hline No & 165 & 76.38 & & \\
\hline Total & 216 & 100 & & \\
\hline
\end{tabular}

GDM - gestational diabetes mellitus

Table-III. Dyslipidemia in GDM cases. $(n=216)$

\begin{tabular}{|c|c|c|c|c|}
\hline \multirow{2}{*}{ Categories } & \multicolumn{4}{|c|}{ Aip Risk Score } \\
\hline & Frequency & $\%$ & $\mathrm{X}^{2}$-Value & P-Value \\
\hline Low risk (-0.3-0.1) & 170 & 78.7 & \multirow{4}{*}{678.0} & \multirow{4}{*}{0.0001} \\
\hline Medium risk (0.1-0.24) & 29 & 13.41 & & \\
\hline High risk (>0.24) & 17 & 7.87 & & \\
\hline Total & 216 & 100 & & \\
\hline
\end{tabular}

Table-IV. Atherogenic index of plasma.

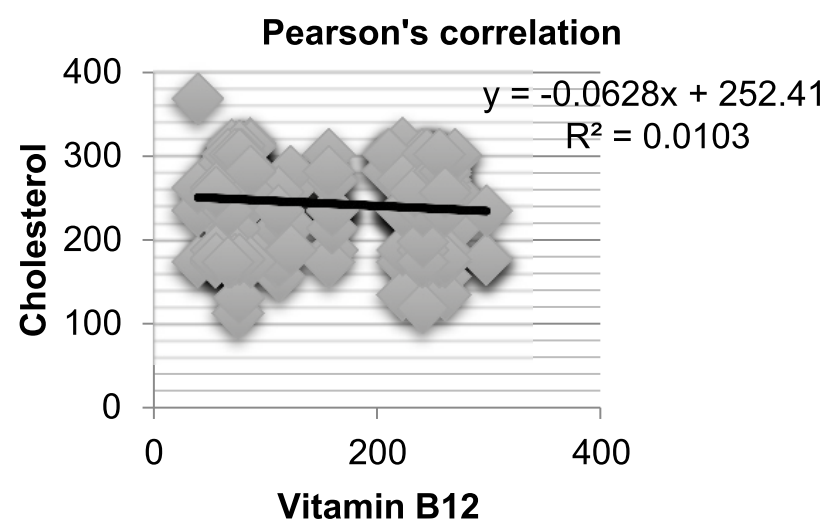

Figure-1. Curve show negative correlation of cholesterol and vitamin $B_{12}(r=-0.25, P=0.02)$

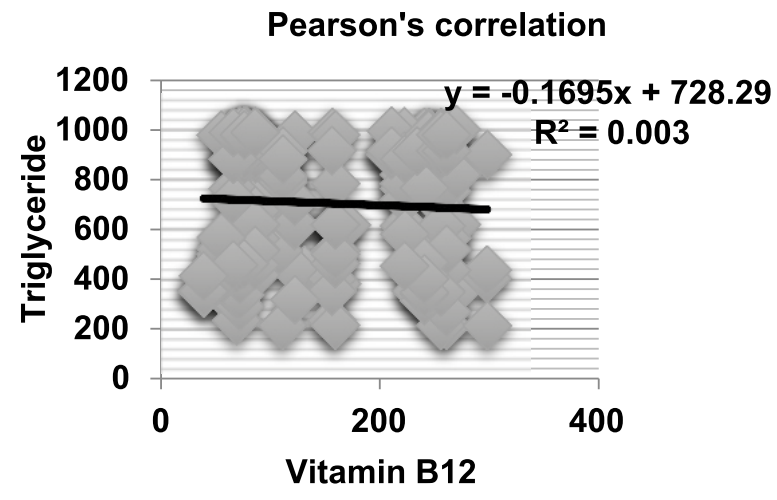

Figure-2. Curve shows negative correlation of triglycerides and vitamin $B_{12}(r=-0.23, P=0.02)$

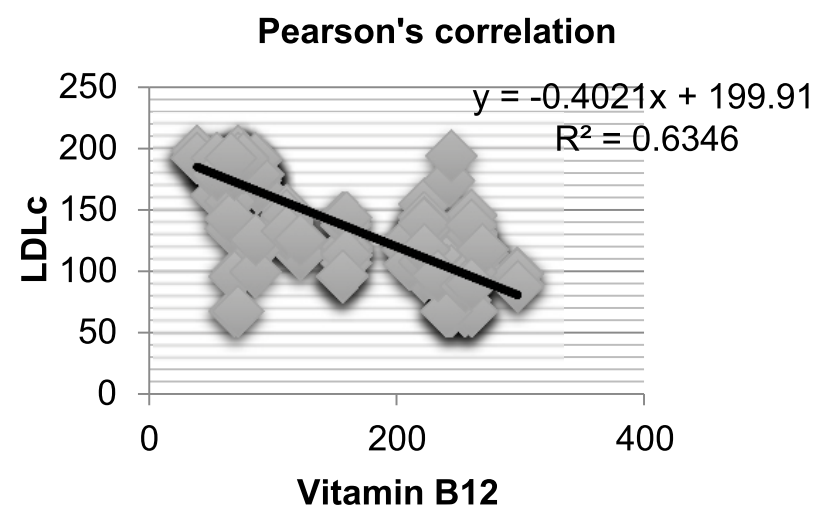

Figure-3. Curve shows negative correlation of LDLC and vitamin $B_{12}(r=-0.79, P=0.0001)$

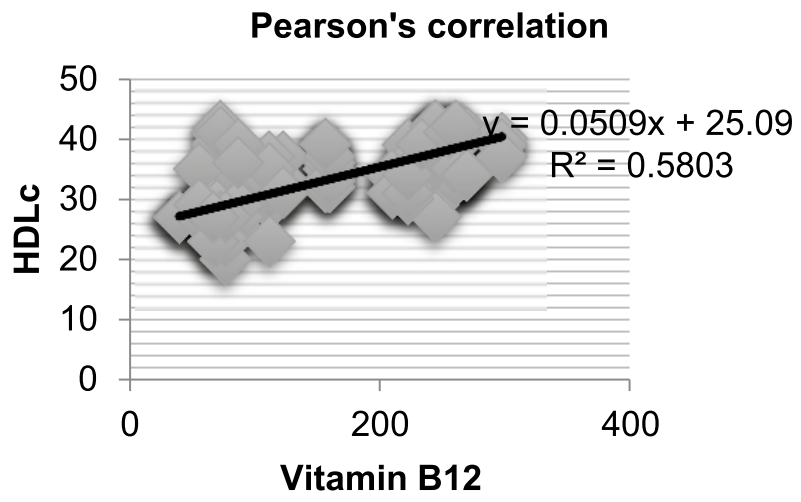

Figure-4. Curve shows positive correlation of HDLC and vitamin $B_{12}(r=0.76, P=0.0001)$

\section{DISCUSSION}

Gestational Diabetes mellitus is a grave complication of pregnancy induced hormonal imbalance in peculiar ethnicity and varies in geographical areas. Present observational is the first reporting on the frequency of dyslipidemia 
and atherogenic tendency (AIP) in vitamin B12 deficient GDM cases. We found vitamin $B_{12}$ deficiency and Dyslipidemia were noted in 151 (69.9\%) and 165 (23.61\%) and high and medium risk AIP was noted in 17 (7.87\%) and 29 (13.4\%) of GDM cases $(P=0.0001)$. The findings are consistent with previous studies. ${ }^{5,7}$ Nutritional deficiency of vitamin B12 is highly prevalent in the Pakistan ${ }^{11,12}$ and its deficiency in GDM may complicate the health of pregnant mothers and babies equally. ${ }^{11,13}$ The present study highlights the issue of pregnant women suffering GDM and concomitant vitamin B12 deficiency, dyslipidemia and atherogenic tendency. The findings are in agreement with previous studies. ${ }^{13,14}$

Deficiency of vitamin B12 in pregnant mothers has been reported previuosly. ${ }^{15,16} \mathrm{~A}$ previous study reported occurrence of insulin resistance and increased adiposity in vitamin B12 deficient pregnant female. ${ }^{16}$ The study concluded the risk of GDM is doubled in vitamin B12 deficient pregnant female. Our findings are supported by both above studies. ${ }^{15,16}$ In present study vitamin $B_{12}$ deficiency was found in $69.9 \%$ of total study sample that is in agreement with a previous study ${ }^{17}$ that found $67 \%$ vitamin B12 deficiency in pregnant female. Deficiency of vitamin B12 deficiency is already highlighted by previous studies. ${ }^{11,12}$ Nutritional deficiencies are prevalent in the developing countries and similar is scenario of Pakistan. ${ }^{11}$ However, it is very astonishing the vitamin B12 deficiency has been reported in pregnant women of developed country ${ }^{18}$, whose food intake is labeled as balanced diet. In present study we noted dyslipidemia in the 165 (23.61\%). High and medium risk AIP was noted in 17 (7.87\%) and $29(13.4 \%)$ of GDM cases $(P=0.0001)$ in present study. Finding of AIP is first analyzed in GDM cases from our institute and is incomparable. The present study noted vitamin $B_{12}$ proved negative TC $(r=-0.25, P=0.02)$, TAG $(r=-0.23$, $P=0.02)$, LDLC $(r=-0.79, P=0.0001)$ and positive correlation with HDLc $(r=0.76, P=0.0001)$. The finding is in agreement with a previous report. ${ }^{18}$ Vitamin B12 deficiency in GDM cases has been reported by a previous study ${ }^{19}$ from UK and further added the risk of GDM is increased 2.59 time in vitamin B12 deficient pregnant female.
We conclude the pregnant female suffering from gestational diabetes mellitus need utmost monitoring of vitamin B12 screening and supplementation to prevent grave complications of dyslipidemia and atherogenicity. The present research is an observational study conducted with small sample size with confounding factor of nutritional deficiency, hence finding is not justified to generalize to other geographical areas.

\section{CONCLUSION}

We found increased frequency of cases vitamin $\mathrm{B}_{12}$ deficiency and dyslipidemia in of GDM cases respectively. High and medium risk of atherogenicity (AIP) was increased GDM cases. Vitamin B12 shows negative correlation with total cholesterol, triglycerides, and low density lipoproteins (LDLC). Vitamin B12 screening may be adapted as routing screening test in gestational diabetes mellitus to prevent grave complications in mothers and their babies.

\section{Copyright $@ 08$ Dec, 2021.}

\section{REFERENCES}

1. Muzaffar H, Chaudhry S, Sadaf N, Hussain R. Efficacy and safety of metformin in gestational diabetes mellitus. Pak J Med Dent 2020; 9 (04): 33-39.

2. Yousuf S, Zafar M, Tabassum A, Mazhar SB. Exercise and its effects on gestational diabetes mellitus and related parameters. Pak J Med Dent 2020; 9 (03): 3236.

3. Adaikalakoteswari A, Finer $S$, Voyias PD, Mc Carthy $\mathrm{CM}$, Vatish M, Moore J, et al. Vitamin B12 insufficiency induces cholesterol biosynthesis by limiting s-adenosylmethionine and modulating the methylation of SREBF1 and LDLR genes. Clinical Epigenetics 2015; 7:1-14.

4. Narindrarangkura P, BosI W, Rangsin R, Hatthachote $P$. Prevalence of dyslipidemia associated with complications in diabetic patients: A nationwide study in Thailand. BMC Lipids Health Dis 2019; 18(90):1-8.

5. Adaikalakoteswari $\mathrm{A}$, Jayashri $\mathrm{R}$, Sukumar $\mathrm{N}$, Venkataraman $\mathrm{H}$, Pradeepa $\mathrm{R}$, Gokulakrishnan K. Vitamin B12 deficiency is associated with adverse lipid profile in Europeans and Indians with type 2 diabetes. BMC Cardiovasc Diabetol 2014; 13(129):1-7. 
6. Yajnik CS. Early life origins of insulin resistance and type 2 diabetes in India and other Asian countries. J Nutr 2014; 134:205-210.

7. Sukumar N, Venkataraman $\mathrm{H}$, Wilson S, Goljan I, Selvamoni S, Patel V. Vitamin B12 status among pregnant women in the UK and its association with obesity and gestational diabetes nutrients 2016, 8 , 768;1-10.

8. Riddle MC. American diaebtes association standards of medical care in diaebtes 2020. Diabet Care January 2020; 43 (1):1-224.

9. Mindiola AL, Fernandez HM, Arciniegas DR, Regino WO. Vitamin B12 deficiency associated with consumption of proton pump inhibitors. Rev Colomb Gastroenterol 2017; 32 (3): 197-201.

10. Lee MJ, Park JT, Han SH, Kim YL, Kim YS, Yang CW, et al. The atherogenic index of plasma and the risk of mortality in incident dialysis patients: Results from a nationwide prospective cohort in Korea. PLOS ONE 2017; 12 (5): e0177499.

11. Fatima SS, Rehman R, Alam F, Madhani S, Chaudhry $B$, Khan TA. Gestational diabetes mellitus and predisposing factors. J Pak Med Assoc 2017; 67: 2615.

12. Subhadra S, Bhagwan SJ, Manisha Y, Usha BR, Sunesh K, Mukhopadhyay AK. Cross-sectional study of nutritional markers in pregnancy. Indian $\mathrm{J}$ Endo Metab 2016; 20 (6) 825-830.
13. Riaz M, Shaikh F, Fawwa A, Hakeem R, Shera AS, Hitman GA, et al. Maternal nutrition during early pregnancy and cardiometabolic status of neonates at birth. J Diabet Res 2018; Article ID 7382946:1- 8.

14. Finkelstein JL, Guillet G, Pressman EK, Fothergill A, Guetterman HM, Kent TR, O'Brien KO. Vitamin B12 Status in Pregnant Adolescents and Their Infants. Nutrients 2019; 11 (397): 1-16

15. Stewart CP, Christian P, Schulze KJ, Arguello M, LeClerq SC, Khatry SK, West KP, Jr. Low maternal vitamin B-12 status is associated with offspring insulin resistance regardless of antenatal micronutrient supplementation in rural Nepal. J Nutr 2011; 141:19127.

16. Krishnaveni G, Hill J, Veena S, Bhat D, Wills A, Karat $\mathrm{C}$, et al. Low plasma vitamin B12 in pregnancy is associated with gestational 'diabesity'and later diabetes. Diabetologia 2009; 52: 2350-8.

17. Butt A, Malik U, Waheed K, Khanum A, Firdous S, Ejaz $\mathrm{S}$, et al. Low serum cobalamin is a risk factor for gestational diabetes. Pakistan J Zool 2017; 49(6):19631968.

18. Knight BA, Shields BM, Brook A, Hill A, Bhat DS, Hattersley AT, Yajnik CS. Lower circulating $B 12$ is associated with higher obesity and insulin resistance during pregnancy in a non-diabetic white British population. PLoS One 2015; 10: e0135268.

19. Sukumar N, Venkataraman H, Wilson S, Goljan I, Selvamoni S, Patel V, et al. Vitamin B12 status among pregnant women in the UK and its association with obesity and gestational diabetes. Nutrients 2016; 8 : 768.

\begin{tabular}{|c|c|c|c|}
\hline \multicolumn{4}{|c|}{ AUTHORSHIP AND CONTRIBUTION DECLARATION } \\
\hline No. & Author(s) Full Name & Contribution to the paper & Author(s) Signature \\
\hline 1 & Shehmeen Khan & $\begin{array}{l}\text { Conception and design, Statisti- } \\
\text { cal expertise, Critical revision. }\end{array}$ & ope \\
\hline 2 & Sabreena Abbas & $\begin{array}{l}\text { Critical revision of the article for } \\
\text { important intellectual content. }\end{array}$ & Simsingin \\
\hline 3 & Afsheen Abro & $\begin{array}{l}\text { Critical revision of the article for } \\
\text { important intellectual content. }\end{array}$ & St- \\
\hline 4 & Beenish Ghafar Memon & $\begin{array}{l}\text { Critical revision of the article for } \\
\text { important intellectual content. }\end{array}$ & (390) \\
\hline 5 & Farhat Sultana & $\begin{array}{l}\text { Data collection, Drafting of the } \\
\text { article. }\end{array}$ & \\
\hline 6 & Hafsa Usman Shaikh & $\begin{array}{l}\text { Data collection, Drafting of the } \\
\text { article. }\end{array}$ & $w$ \\
\hline
\end{tabular}

